International Journal of Modern Physics E

(C) World Scientific Publishing Company

\title{
SIMULATION OF JET QUENCHING OBSERVABLES IN HEAVY ION COLLISIONS AT THE LHC
}

\author{
CHRISTOF ROLAND* \\ Laboratory for Nuclear Science, Massachussetts Institute of Technology \\ 77 Massachusetts Avenue, Cambridge, MA 02139-4307, USA \\ christof.roland@cern.ch \\ GÁBOR I. VERES, KRISZTIÁN KRAJCZÁR \\ Department of Atomic Physics, Eötvös Loránd University \\ Pázmány Péter sétány 1/A, Budapest, H-1117, Hungary \\ vg@ludens.elte.hu,nirnaeth@monornet.hu \\ for the CMS Heavy Ion Group \\ Received (received date) \\ Revised (revised date)
}

\begin{abstract}
Large transverse momentum jets provide unique tools to study dense QCD matter in high-energy heavy-ion collisions. Results from RHIC on suppression of high transverse momentum particles in $\mathrm{Au}+\mathrm{Au}$ collisions indicate a significant energy loss of leading partons in the dense and strongly interacting matter formed in these collisions. The LHC will collide $\mathrm{Pb}$ ions at $\sqrt{s_{N N}}=5500 \mathrm{GeV}$, where the cross section of hard scattering will increase dramatically. Large production rates, the large acceptance of the CMS calorimeters and tracking system, combined with the capability of triggering on jets, will extend the transverse momentum reach of charged particle spectra and nuclear modification factors up to $p_{T}>200 \mathrm{GeV} / c$.
\end{abstract}

\section{Introduction}

The abundance of high $Q^{2}$ processes at LHC energies will provide large samples of high $E_{\mathrm{T}}$ jets, large $p_{\mathrm{T}}$ hadrons, and jets produced opposite to gauge bosons $\left(\gamma^{\star}, Z\right) ! 1$ The strong interest in these observables in heavy-ion collisions stems from the concept that high $E_{\mathrm{T}}$ quark and gluon jets can be used to probe the hot and dense medium produced in the collision, because they are affected by the properties of the medium as they propagate through this dense environment. Partons with high transverse momentum are predicted to suffer radiative and collisional energy loss in the created medium, suppressing the yield of jets and particles found with high transverse energy in a heavy-ion collision, compared to the $\mathrm{p}+\mathrm{p}$ collision case (see e.g. 2 ).

*address: CERN, Switzerland 
Early results obtained at RHIC indeed showed suppression of the hadron yield at $p_{\mathrm{T}}>3 \mathrm{GeV} / c$ and the disappearance of back-to-back correlations of high- $p_{\mathrm{T}}$ particles! 3 These indirect measurements of jet properties suggest a significant inmedium energy loss of fast partons, which will be experimentally accessible at the LHC also by observing fully formed and reconstructed jets.

The performance of the CMS detector for $\mathrm{Pb}+\mathrm{Pb}$ events was extensively studied in full simulations with realistic assumptions for particle multiplicity, jet and hadron spectra. 4 The charged particle reconstruction capabilities using the CMS Silicon Tracking System are evaluated using a full detector simulation, assuming a charged particle density in central $\mathrm{Pb}+\mathrm{Pb}$ collisions of $\mathrm{d} N_{\mathrm{ch}} / \mathrm{d} y=3200$. In this high multiplicity environment, an algorithmic tracking efficiency of about $80 \%$ is achieved, with less than $5 \%$ fake track rate for $p_{\mathrm{T}}>1 \mathrm{GeV} / c$ and excellent momentum resolution, $\Delta p_{\mathrm{T}} / p_{\mathrm{T}}<1.5 \%$ (for $p_{\mathrm{T}}<100 \mathrm{GeV} / c$ ). 5 .

The High Level Trigger of the CMS data acquisition system is sufficiently powerful to allow the inspection of all minimum bias $\mathrm{Pb}+\mathrm{Pb}$ events individually, where the full event information will be available for the trigger decision. ${ }^{6}$ Jets are reconstructed in the calorimeters using an iterative cone algorithm, which is modified to subtract the underlying soft background, and can be included in the HLT. The lower limit of transverse energy needed for efficient and clean reconstruction is about 50 $\mathrm{GeV}$. The energy resolution for jets with $100 \mathrm{GeV}$ transverse energy at $\eta \approx 0$ is about $16 \% !$

\section{Nuclear Modification Factor}

The nuclear modification factor, $R_{A A}$, and the central to peripheral ratio, $R_{C P}$, provide quantitative information on the amount and energy-dependence of the energyloss of hard-scattered partons that traverse the medium created in the heavy-ion collision. They are defined as:

$$
R_{A A}=\frac{\sigma_{p p}^{\text {inel }}}{\left\langle N_{\text {coll }}\right\rangle} \frac{\mathrm{d}^{2} N_{A A} / \mathrm{d} p_{\mathrm{T}} \mathrm{d} \eta}{\mathrm{d}^{2} \sigma_{p p} / \mathrm{d} p_{\mathrm{T}} \mathrm{d} \eta} \quad \text { and } \quad R_{C P}=\frac{\left\langle N_{\text {coll }}^{\text {periph }}\right\rangle}{\left\langle N_{\text {coll }}^{\text {central }}\right\rangle} \frac{\mathrm{d}^{2} N_{A A}^{\text {central }} / \mathrm{d} p_{\mathrm{T}} \mathrm{d} \eta}{\mathrm{d}^{2} N_{A A}^{\text {periph }} / \mathrm{d} p_{\mathrm{T}} d \eta},
$$

where $\left\langle N_{\text {coll }}\right\rangle$ is the number of binary nucleon-nucleon collisions in the geometrical Glauber picture, averaged over the events that belong to a given centrality class.

$R_{A A}$ quantifies the suppression (or enhancement) of hadron production with respect to $\mathrm{p}+\mathrm{p}$ collisions. The invariant cross section of charged particles at high $p_{\mathrm{T}}$ is expected to scale with $\left\langle N_{\text {coll }}\right\rangle$ if no nuclear effects take place, in which case the value of $R_{A A}$ at high $p_{\mathrm{T}}$ would be unity. $R_{C P}$ does not require a $\mathrm{p}+\mathrm{p}$ reference, as it compares central and peripheral heavy-ion collisions. It is not equivalent to $R_{A A}$, since even the most peripheral heavy-ion collisions are influenced by nuclear effects.

The $R_{A A}\left(p_{T}\right)$ and $R_{C P}\left(p_{T}\right)$ for charged hadrons thus provide important information on the properties of the created medium, like the initial gluon rapidity density, $\mathrm{d} N_{g} / \mathrm{d} y$, or the transport coefficient, $\langle\hat{q}\rangle, \underline{2}$ 


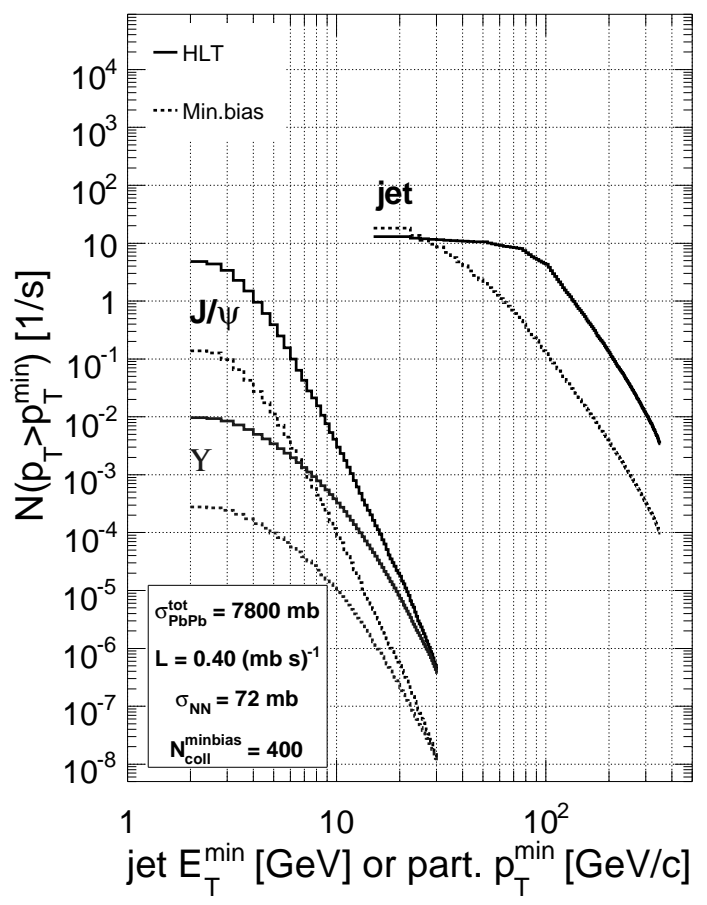

Fig. 1. Event rate of various hard processes stored on tape above a certain $E_{\mathrm{T}}\left(p_{\mathrm{T}}\right)$ at the design $\mathrm{Pb}+\mathrm{Pb}$ luminosity. The minimum bias and the HLT data taking modes are compared.

\section{Triggering on Jets}

For both $R_{A A}$ and $R_{C P}$, triggering on jets will be essential to extend the measurable $p_{\mathrm{T}}$ range. Using the CMS calorimeter towers, it is possible to quickly reconstruct these energetic jets with a good energy resolution. The jet reconstruction algorithm can be included in the High Level Trigger, and will record events containing a high energy jet with high efficiency $[6]$ The increase in the number of recorded jets using the jet trigger is illustrated in Fig. 1.

Charged hadrons at $p_{\mathrm{T}}>20-30 \mathrm{GeV} / c$ originate mainly from the fragmentation of high $E_{\mathrm{T}}$ jets: they are typically the leading hadrons of the energetic jets. The jet trigger is useful to collect sufficiently large jet statistics to study fragmentation functions, jet correlations, and other observables, among which we will present here the charged particle nuclear modification factors. To provide adequate and fast Monte Carlo tools to simulate jet quenching, the Monte Carlo event generator HYDJET (HYDrodynamics plus JETs) has been developed and is used to produce heavy-ion collisions at LHC energies $\frac{418}{6}$ Final state particles in nuclear collisions from HYDJET are obtained as a superposition of soft hydro-type particle production and multiple hard parton-parton collisions. 

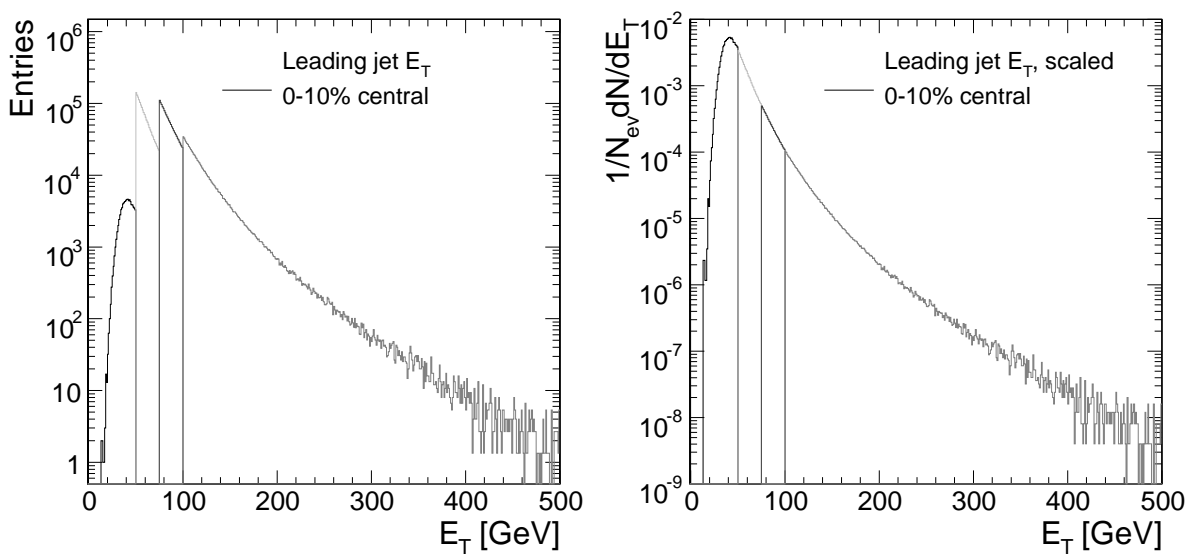

Fig. 2. Left panel: leading jet $E_{\mathrm{T}}$ distributions for minimum bias (black), and triggered simulated data samples, with $E_{\mathrm{T}}$ thresholds of 50, 75 and $100 \mathrm{GeV}$. Right panel: the same distributions sliced into the $0-50,50-75,75-100$ and $100-300 \mathrm{GeV}$ intervals, and scaled by the appropriate factors to get back the non-triggered distribution, with significantly higher statistics.

\section{Results}

Within the $15 \%$ of the full bandwidth assigned to minimum bias events, 13.5 million events are expected to be taken in one month. The jet triggers with 50, 75 and $100 \mathrm{GeV} E_{T}$ thresholds will be able to sample $0.35,1.9$ and 3.9 billion events, respectively. It is possible to generate the amount of minimum bias events with our generator-level tools. However, triggering enhances the number of jets at high $E_{\mathrm{T}}$ by more than two orders of magnitude. Thus, instead of generating one hundred times more minimum bias heavy-ion events to conduct our study, we have implemented a "trigger" at the generator level. This way, we only store simulated events which are likely to produce a large $E_{\mathrm{T}}$ jet when the jet finder is run on the calorimeter towers. Thus, more events are sampled than stored and the necessary scaling factors between the different trigger samples are not immediately available.

Figure 2 shows the distribution of the highest $E_{\mathrm{T}}$ jet within $|\eta|<2$ per event, the "leading" jet, for minimum bias (black histogram), and for jet-triggered events with 50, 75 and $100 \mathrm{GeV}$ thresholds (grey histograms). The scaling factors between consecutive data sets are determined by joining them with scaling factors determined by fitting the combined leading jet $E_{T}$ spectrum with a power law in the joining regions. This way, the optimal scaling factors can be determined from the data distribution, without any prior assumptions on the spectrum. This is illustrated in Fig. 3, the left panel shows the data sets before merging while the right panel shows the same data sets after scaling, together with the power law fit. The minimum bias $E_{\mathrm{T}}$ spectrum is recovered with significantly increased statistics at high $E_{\mathrm{T}}$.

Using this method to merge data sets with different thresholds, triggered data 

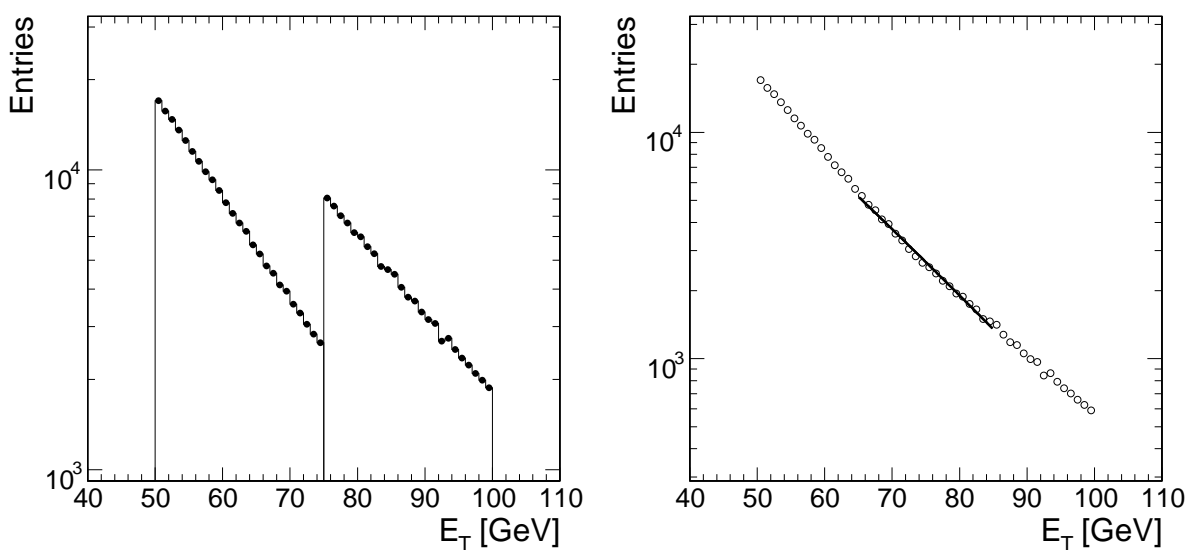

Fig. 3. Left panel: leading jet $E_{\mathrm{T}}$ distributions for two consecutive triggered data samples. Right panel: the same distributions scaled with the factor obtained in the merging procedure.
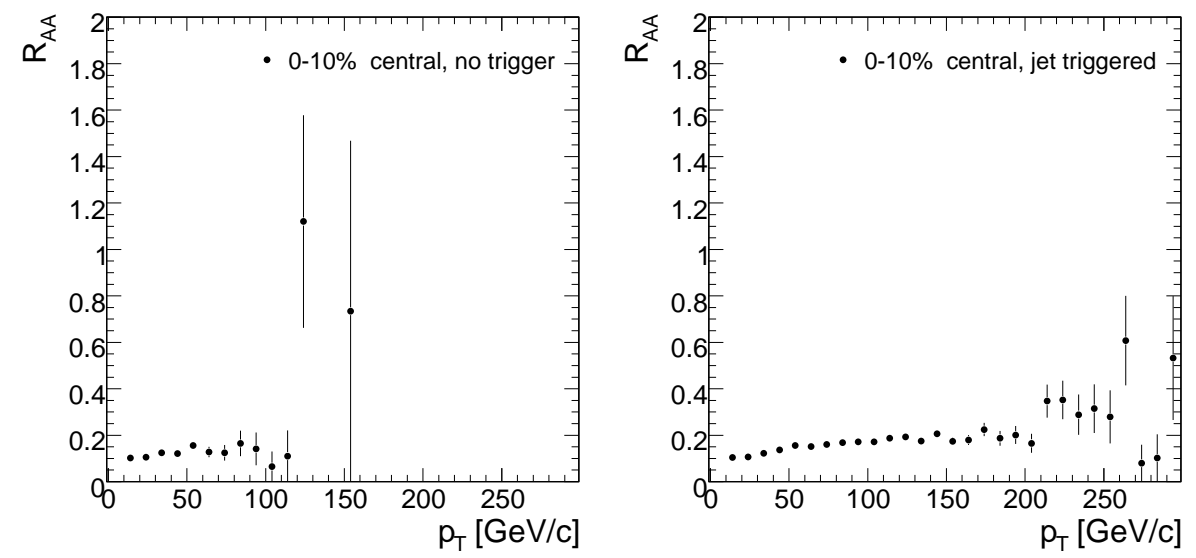

Fig. 4. The nuclear modification factor $R_{A A}$ as a function of $p_{\mathrm{T}}$ for charged particles, for minimum bias data (left) and for data triggered on high- $E_{\mathrm{T}}$ jets (right), for one month of data taking.

sets were generated with the number of jets with $E_{\mathrm{T}}$ above the thresholds expected from one month of $\mathrm{Pb}+\mathrm{Pb}$ data taking at design luminosity. The statistical errors on the charged particle $p_{\mathrm{T}}$ spectrum in the merged data set reflects, hence, the real experimental situation after one month of data taking, with the four different trigger thresholds.

The obtained $R_{A A}$ is shown in Fig. 4. In the present study, the PYTHIA event generator was used to simulate the $\mathrm{p}+\mathrm{p}$ reference for $R_{A A}$. The $R_{C P}$ ratio, which uses peripheral $\mathrm{Pb}+\mathrm{Pb}$ collisions as reference, instead of $\mathrm{p}+\mathrm{p}$ collisions, is shown in Fig. [5 for one month of data taking at nominal luminosity. Comparing the results 

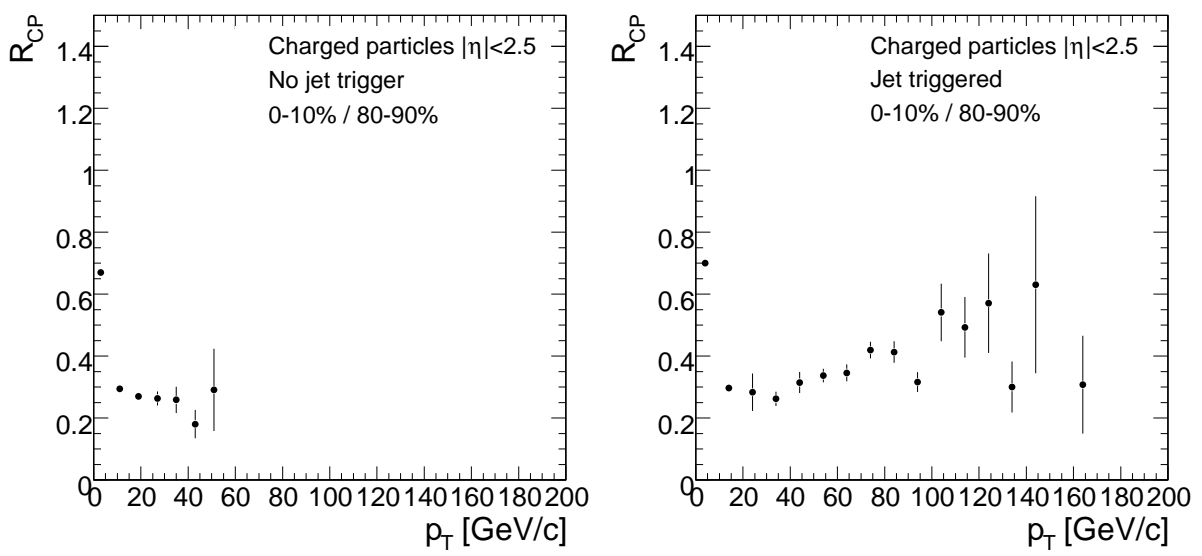

Fig. 5. The central-to-peripheral ratio, $R_{C P}$, as a function of $p_{\mathrm{T}}$, for charged particles, for minimum bias data (left) and for data triggered on high- $E_{\mathrm{T}}$ jets (right), for one month of data taking.

for the minimum bias data (left panels of Figs. 4 and 5 ) to the results for the jet triggered data (right panels), we see that triggering on jets significantly extends the $p_{\mathrm{T}}$ range of $R_{A A}$ and $R_{C P}$, from $\sim 100$ to $\sim 250 \mathrm{GeV} / c$ in $R_{A A}\left(p_{\mathrm{T}}\right)$ and from $\sim 50$ to $\sim 150 \mathrm{GeV} / c$ in $R_{C P}\left(p_{\mathrm{T}}\right)$.

\section{Acknowledgements}

The authors are indebted to M. Ballintijn, C. Loizides, I. P. Lokhtin, S. V. Petrushanko, G. Roland, L. I. Sarycheva, A. M. Snigirev, I. N. Vardanyan, B. Wyslouch, and to the CMS Heavy Ion group for valuable discussions, and to all contributors of the HIROOT analysis tool. Two of us (G.I.V. and K.K.) thanks the support of the Hungarian Scientific Research Fund (T 048898 and F 049823).

\section{References}

1. D. Brandt, LHC Project Report (2000) 450.

2. C. A. Salgado and U. A. Wiedemann, Phys. Rev. D68 (2003) 014008.

3. K. Adcox et al. (PHENIX), Phys. Lett. B561 (2003) 82-92,

B. B. Back et al. (PHOBOS), Phys. Rev. Lett. 91 (2003) 072302,

J. Adams et al. (STAR), Phys. Rev. Lett. 91 (2003) 072304,

B. B. Back et al. (PHOBOS), Phys. Lett. B578 (2004) 297-303,

B. B. Back et al. (PHOBOS), Phys. Rev. Lett. 94 (2005) 082304

4. I. P. Lokhtin and A. M. Snigirev, Eur. Phys. J. C45 (2006) 211.

5. C. Roland, CMS Note 2006/031.

6. M. Ballintijn, C. Loizides and G. Roland, ArXiv:nucl-ex/0702041, "CMS Physics TDR Addendum: High Density QDC with Heavy-Ions", CERN-LHCC-2007, to be submitted

7. O. Kodolova et al., CMS Note 2006/050.

8. http://cern.ch/lokhtin/hydro/hydjet.html 